\title{
MRI-Based Multimodal Approach to the Assessment of Clinical Symptom Severity of Obsessive-Compulsive Disorder
}

\author{
Shin-Eui Park ${ }^{1}$, Byeong-Chae Kim², Jong-Chul Yang ${ }^{3 凶}$, and Gwang-Woo Jeong ${ }^{4 凶}$ \\ ${ }^{1}$ Center for Research Equipment, Korea Basic Science Institute, Cheongju, Republic of Korea \\ ${ }^{2}$ Department of Neurology, Chonnam National University Medical School, Gwangju, Republic of Korea \\ ${ }^{3}$ Department of Psychiatry, Research Institute of Clinical Medicine of Jeonbuk National University, Jeonju, Republic of Korea \\ ${ }^{4}$ Department of Radiology, Chonnam National University Medical School, Gwangju, Republic of Korea
}

Objective This study assessed the associations of the abnormal brain activation and functional connectivity (FC) during memory processing and brain volume alteration in conjunction with psychiatric symptom severity in patients with obsessive-compulsive disorder (OCD).

Methods Twenty-OCD patients and 20-healthy controls (HC) underwent T1-weighted and functional imaging underlying explicit memory task.

Results In memory encoding, OCD patients showed higher activities in right/left (Rt./Lt.) inferior temporal gyrus (ITG), medial prefrontal cortex (MPFC), dorsolateral prefrontal cortex (DLPFC) and anterior cingulate cortex (ACC), compared with HC. In task-based FC, caudate (Cd) was positively connected with DLPFC and ITG in OCD, while HC showed different connectivities of Cd-ACC and Rt.-Lt. ITG. In memory retrieval, only Cd was activated in OCD patients. Cd was positively connected with DLPFC and vmPFC in OCD, but negatively connected between same brain areas in HC. OCD patients showed increased gray matter (GM) volumes of cerebellum, DLPFC, orbitofrontal cortex (OFC), hippocampus, Cd and ITG, and concurrently, increased white matter volumes of DLPFC. In OCD patients, GM volumes of Cd and OFC were positively correlated with HAMA and Y-BOCS. Functional activity changes of Cd in OCD were positively correlated with Y-BOCS.

Conclusion Our findings support to accessing clinical symptom and its severity linked by brain structural deformation and functional abnormality in OCD patients.

Psychiatry Investig 2020;17(8):777-785

Key Words Obsessive-compulsive disorder, Functional connectivity, Functional magnetic resonance imaging,

Voxel-based morphometry, Explicit memory.

\section{INTRODUCTION}

Obsessive-compulsive disorder (OCD) is a common psychiatric disorder characterized by intrusive thoughts, repetitive acts and/or compulsive behaviors with a lifetime prevalence of $2.3 \% .{ }^{1,2}$ Especially, OCD behaviors involve the cognitive dys-

Received: April 6, 2020 Accepted: May 31, 2020

$\triangle$ Correspondence: Gwang-Woo Jeong, MPh, PhD

Department of Radiology, Chonnam National University Medical School, 42 Jebong-ro, Dong-gu, Gwangju 61469, Republic of Korea

Tel: $+82-62-220-5881$, Fax: $+82-62-226-4380$

E-mail: gwjeong100@daum.net

$\triangle$ Correspondence: Jong-Chul Yang, MD, PhD

Department of Psychiatry, Jeonbuk National University Medical School, 20 Geonji-ro, Deokjin-gu, Jeonju 54907, Republic of Korea

Tel: +82-63-250-2580, Fax: +82-63-275-3157

E-mail: yangjc@jbnu.ac.kr

(a) This is an Open Access article distributed under the terms of the Creative Commons Attribution Non-Commercial License (https://creativecommons.org/licenses/by$\mathrm{nc} / 4.0$ ) which permits unrestricted non-commercial use, distribution, and reproduction in any medium, provided the original work is properly cited. function and poor memory. There are many neuro-functional studies $^{3-8}$ on the abnormal brain activity with memory dysfunction, particularly in the strategic memory processing. ${ }^{3.5}$ In general, patients with OCD tend to over-focus on details and miss the larger context, leading to memory impairment. ${ }^{6,7}$

A recent functional connectivity study ${ }^{9-11}$ suggested that the cognitive dysfunction in OCD patients is possibly related to the abnormal neural interactions within brain circuits associated with neurocognitive endophenotypes. Also, brain morphometric studies ${ }^{12,13}$ showed a correlation of the brain volume changes and psychiatric symptom severity. Recently, Moon et al. ${ }^{7}$ used a combined study with functional magnetic resonance imaging (fMRI) and voxel based morphometry (VBM) to demonstrate the identical brain areas showing low functional activities and brain volume reduction. From the knowledge of the previous studies, ${ }^{3,4,6-8,12,13}$ we assumed that altered brain activity and functional connection and morphometric abnormality are closely 
associated with OCD symptom and its severity.

In current study, therefore, we performed the neuroimaging studies on the explicit memory task using MR-based multimodal techniques including fMRI, task-induced functional connectivity and VBM to assess the associations of neurofunctional activation pattern, functional connectivity and morphometric change in connection with clinical symptom severities represented by Yale-Brown Obsessive-Compulsive Scale (Y-BOCS), Hamilton Depression Scale 17 (HAMD-17) and Hamilton Anxiety Scale(HAMA) in patients with OCD.

\section{METHODS}

\section{Subjects}

Forty right-handed subjects included twenty patients with OCD (mean age, 29.4 \pm 9.9 years), who are diagnosed by a psychiatrist using the DSM-IV-TR, ${ }^{14}$ and 20 healthy controls (mean age, $29.7 \pm 8.3$ years) with no history of neurological or psychiatric illness. The duration of patient's illness was $5.8 \pm 4.7$ years (Table 1). Out of 20 patients, eight patients received a single psychotropic medication including escitalopram $(n=6)$ and fluvoxamine $(\mathrm{n}=2)$, while twelve patients received multiple psychotropic medications including anxiolytics: alprazolam $(n=5)$, buspirone $(n=1)$ and clonazepam $(n=2)$; antidepressants: escitalopram $(n=11)$, and fluvoxamine $(n=1)$; atypical antipsychotics: amisulpride $(n=3)$ and paliperidone $(n=1)$. Written informed consents were obtained from all the participants before the study. The Institutional Review Board (IRB) of Chonbuk National University Hospital in Korea approved the study protocol (IRB File No: 2016-05-024, 2019-07-016).

\section{Clinical interviews}

All the participants underwent the clinical interviews using Yale-Brown Obsessive-Compulsive Scale (Y-BOCS; ten items with five level scale, cutoff score $>7$ ), Hamilton Depres-

Table 1. Characteristics of patients with OCD and healthy controls

\begin{tabular}{lccc}
\hline & $\begin{array}{c}\text { OCD } \\
(\mathrm{N}=20)\end{array}$ & $\begin{array}{c}\text { Controls } \\
(\mathrm{N}=20)\end{array}$ & p-value \\
\hline Age (years) & $29.4 \pm 9.9$ & $29.7 \pm 8.3$ & $0.533^{*}$ \\
Sex (male/female) & $15 / 5$ & $14 / 6$ & $0.723^{\dagger}$ \\
Handness (\% right) & 100 & 100 & 1.000 \\
Education (years) & $14.6 \pm 1.9$ & $14.7 \pm 2.5$ & $0.780^{*}$ \\
Duration of illness (years) & $5.8 \pm 4.7$ & - & - \\
Y-BOCS & $26.1 \pm 3.3$ & $0.0 \pm 0.0$ & $<0.0001^{*}$ \\
HAMA & $11.1 \pm 7.4$ & $0.6 \pm 0.9$ & $<0.0001^{*}$ \\
HAMD-17 & $7.6 \pm 1.9$ & $0.3 \pm 0.6$ & $<0.0001^{*}$ \\
\hline
\end{tabular}

${ }^{*}$ Mann Whitney U test, ${ }^{\dagger}$ chi-square test. Y-BOCS: Yale-Brown Obsessive Compulsive Scale, HAMA: Hamilton Anxiety Scale, HAMD-17: Hamilton Depression Scale 17 sion Scale 17 (HAMD-17; 8 items with five-level scale and nine items with three level scale, cutoff score >7) and Hamilton Anxiety Scale (HAMA; 14 items with a five-level scale, cutoff score $>14$ ). The difference of symptom severity in both groups was analyzed by Mann-Whitney U test.

\section{Paradigm for brain activation}

The activation paradigm consisted of the following cycle: rest (14s), first encoding (18s), rest (14s), first retrieval (18s), rest (14s), second encoding (18s), rest (14s), second retrieval (18s), and rest (14s) (Figure 1). In the rest periods, two fixation crosses were displayed. In each encoding period, six different two-syllable words were displayed for 3 s per each slice. In the retrieval periods, the words mixed with old (showed in encoding period) and new were randomly presented. The participants were instructed to press the button if the presented word is old word. All of the words were displayed on a computer monitor using Superlab Pro software (Cedrus Co., San Pedro, CA, USA).

\section{Data acquisition}

MRI scanning was performed on a 3 Tesla Magnetom Verio MR Scanner (Siemens Medical Solutions, Erlangen, Germany) with an 8-channel birdcage type of head coil. Anatomical T1weighted images were acquired using a three-dimensional magnetization-prepared rapid acquisition gradient-echo sequence with repetition time (TR)/echo time (TE)=1,900/2.35 $\mathrm{ms}$, field-of-view $(\mathrm{FOV})=22 \times 22 \mathrm{~cm}^{2}$, matrix size $=256 \times 256$, number of excitation $(\mathrm{NEX})=1$ and slice thickness $=1 \mathrm{~mm}$. Functional images were acquired from a total of 25 axial slices parallel to an anterior commissure to posterior commissure line using a gradient-echo echo planner image (GRE-EPI) with the follow parameters: TR/TE $=2,000 / 30 \mathrm{~ms}$, flip angle $=$ $90^{\circ}, \mathrm{FOV}=22 \times 22 \mathrm{~cm}^{2}$, metrix size $=64 \times 64, \mathrm{NEX}=1$, and slice thickness $=5 \mathrm{~mm}$ without a slice gap.

\section{Data processing and statistical analysis}

\section{Functional brain mapping}

Functional imaging data was analyzed using the SPM12 software (Wellcome Department of Cognitive Neurology, London,

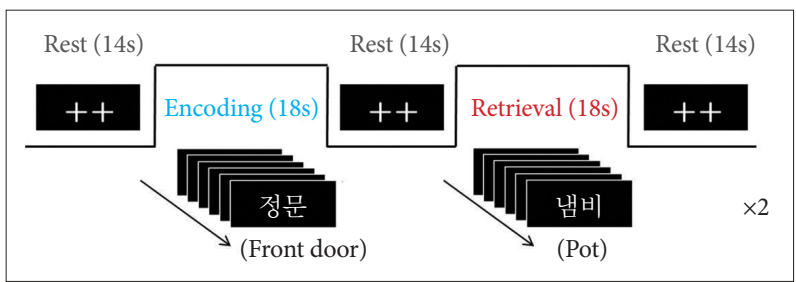

Figure 1. fMRI paradigm for brain activation in memory encoding and retrieval task. 
UK). The image data were reconstructed using am optimized protocol described in our previous study. ${ }^{15}$ To analyze the group difference of brain activation underlying memory task, a twosample t-test was performed in the encoding and retrieval condition ( $p<0.001$ with a cluster size of $>30$ contiguous voxels).

\section{Functional connectivity}

The fMRI and structural MRI data were processed using CONN-fMRI FC toolbox (ver.17e) with the SPM12. First, all functional images were realigned and unwrapped, slice-timing corrected, coregistered with structural data, spatially normalized into the standard MNI space (Montreal Neurological Institute, Canada), outlier detected and smoothed using a Gaussian kernel with a full-with-at-half maximum (FWHM) of $8 \mathrm{~mm}$. Structural data were segmented into the gray matter (GM), white matter (WM) and cerebrospinal fluid (CSF), and normalized to remove temporal confounding factors. The effect of nuisance covariates, including fluctuations in fMRI signals from WM, CSF and their derivatives, as well as realignment parameter noise, was reduced by using anatomical component-based noise correction method (aCompCor).$^{16}$ Band-pass filtering were performed with a frequency window of $0.01-0.1 \mathrm{~Hz}$. To analyze the functional connectivity, we conducted a region of interest (ROI)-to-ROI FC analysis. We specified six spherical clusters with $5 \mathrm{~mm}$ diameters and peak coordinates based on fMRI results. The ROIs were located in the inferior temporal cortex (ITC; right: $\mathrm{x}=48, \mathrm{y}=12, \mathrm{z}=-40$, left: $-44,-18,-22$ ), medial prefrontal cortex (MPFC: -10, 54, 20), dorsolateral prefrontal cortex (DLPFC: -28, 38, 20), anterior cingulate cortex (ACC: 14, 34, 22) and caudate (Cd: 6, 8, 2 ). General linear model (GLM) was used to measure the correlation of oxygen-level-dependent (BOLD) time series between the seed area and ROIs observed in fMRI. Significant connections were identified by calculating the uncorrected two-sided p-value $<0.005$.

\section{Brain volumetric difference}

The structural image data were post-processed using the SPM8 program with DARTEL-based VBM analysis following the procedure described in our previous study. ${ }^{12}$ Alterations in GM and WM volumes in patients with OCD and healthy controls were assessed by the independent two-sample t-test with multiple comparisons using family wise error (FEW at $\mathrm{p}<0.05$ ).

\section{RESULTS}

\section{Demographic characteristics and symptom severity}

The demographic information and symptom severity of patient with OCD and healthy controls are described in Table 1. There are no differences between the two groups in terms of age, gender distribution, and length of education. Compared with controls, OCD patients showed higher scores in all symptom scores.
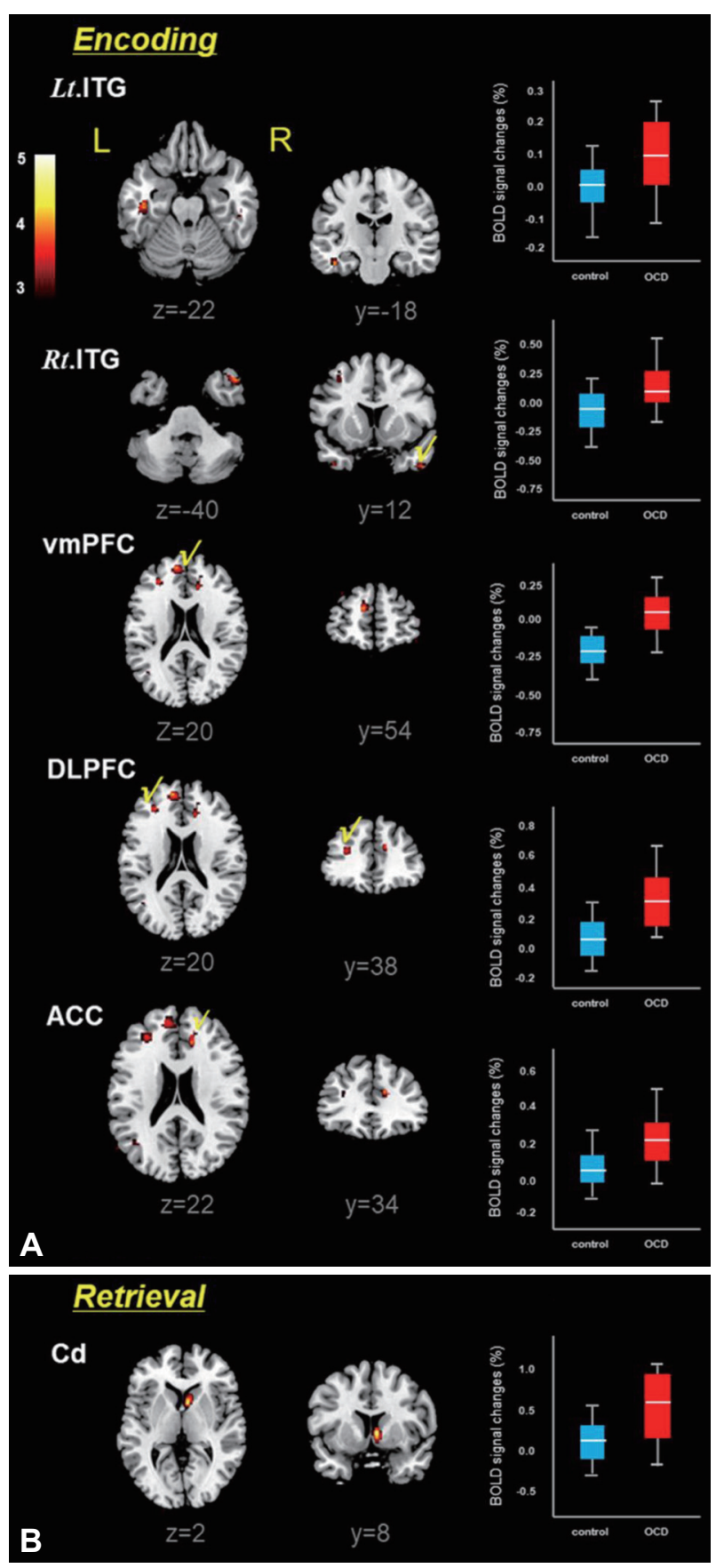

Figure 2. Brain areas predominantly activated in patients with $O C D$ relative to healthy controls during memory task: encoding epoch $(A)$ and retrieval epoch $(B)$, resulting from the two-sample t-test (uncorr., $p<0.001$ ). Box-plots show the medians, interquartile ranges, highest and lowest values for BOLD signal intensities of corresponding brain areas. The color bar represents the t-value. Rt./Lt. ITG: right/left inferior temporal gyrus, vmPFC: ventromedial prefrontal cortex, DLPFC: dorsolateral prefrontal cortex, ACC: anterior cingulate cortex, Cd: caudate, L: left; R: right. 
Table 2. Predominantly activated brain areas during the course of encoding and retrieval in explicit memory task: patient with OCD vs. healthy controls $(p<0.001, t=3.32)$

\begin{tabular}{|c|c|c|c|c|c|c|c|c|c|c|}
\hline \multirow{3}{*}{ Brain areas } & \multicolumn{5}{|c|}{ OCD $>$ controls } & \multicolumn{5}{|c|}{ OCD $<$ controls } \\
\hline & \multirow{2}{*}{$\begin{array}{c}\text { Maximum } \\
\text { t-value }\end{array}$} & \multicolumn{3}{|c|}{ MNI coordinates } & \multirow{2}{*}{$\begin{array}{c}\text { Voxels in } \\
\text { cluster }\end{array}$} & \multirow{2}{*}{$\begin{array}{c}\text { Maximum } \\
\text { t-value }\end{array}$} & \multicolumn{3}{|c|}{ MNI coordinates } & \multirow{2}{*}{$\begin{array}{c}\text { Voxels in } \\
\text { cluster }\end{array}$} \\
\hline & & $\mathrm{x}$ & $\mathrm{y}$ & $\mathrm{z}$ & & & $\mathrm{x}$ & $\mathrm{y}$ & $\mathrm{z}$ & \\
\hline \multicolumn{11}{|l|}{ Encoding } \\
\hline Left inferior temporal gyrus (Lt.ITG) & 4.31 & -44 & -18 & -22 & 63 & - & - & - & - & - \\
\hline Right inferior temporal gyrus (Rt.ITG) & 4.22 & 48 & 12 & -40 & 43 & - & - & - & - & - \\
\hline ventromedial prefrontal cortex (vmPFC) & 4.03 & -10 & 54 & 20 & 53 & - & - & - & - & - \\
\hline Dorsolateral prefrontal cortex (DLPFC) & 3.86 & -28 & 38 & 20 & 31 & - & - & - & - & - \\
\hline Anterior cingulate cortex (ACC) & 3.46 & 14 & 34 & 22 & 33 & - & - & - & - & - \\
\hline \multicolumn{11}{|l|}{ Retrieval } \\
\hline Caudata (Cd) & 5.52 & 6 & 8 & 2 & 160 & - & - & - & - & - \\
\hline
\end{tabular}

OCD: obsessive-compulsive disorder

\section{Differential brain activation patterns}

Differential brain activation patterns for the encoding and retrieval epochs between OCD patients and healthy controls are shown at Figure 2. Also, the predominantly activated brain areas in the two groups are summarized in Table 2 . Compared with healthy controls, OCD patients showed higher activities in the right/left (Rt/Lt) inferior temporal gyrus (ITG), medial prefrontal cortex (MPFC), dorsolateral prefrontal cortex (DLPFC) and anterior cingulate cortex (ACC) during the memory encoding epoch $(\mathrm{p}<0.001)$. In the memory retrieval period, only the caudate $(\mathrm{Cd})$ was predominantly activated in patient with OCD $(\mathrm{p}<0.001)$. It should be noted that OCD patients show none of the specific brain areas with lower activities relative to healthy controls (Figure 2).

\section{Correlation of BOLD signal changes with symptom severity}

In patients with $\mathrm{OCD}$, the BOLD signal change of the $\mathrm{Cd}$ was positively correlated with Y-BOCS scores $(r=0.478$, $\mathrm{p}=0.033$ ) (Figure 3). However, no correlation was observed in healthy controls. Pearson's correlation coefficients were satisfied with the critical value as $\mathrm{r}=0.444$ at $\mathrm{p}=0.05$ (two-tailed test).

\section{Functional connectivity}

Task-induced functional connectivity was observed during the explicit memory task. In the encoding period, OCD patients showed positive connectivity in the Cd, DLPFC and ITG, while healthy controls showed positive connectivity of Cd-ACC and Rt. ITG-Lt. ITG ( $\mathrm{p}<0.005)$. In the retrieval period, the Cd, DLPFC and vmPFC are positively connected to each other in OCD, but the same brain areas were negatively connected in healthy controls by contrast $(\mathrm{p}<0.005)$ (Figure 4 , Table 3).

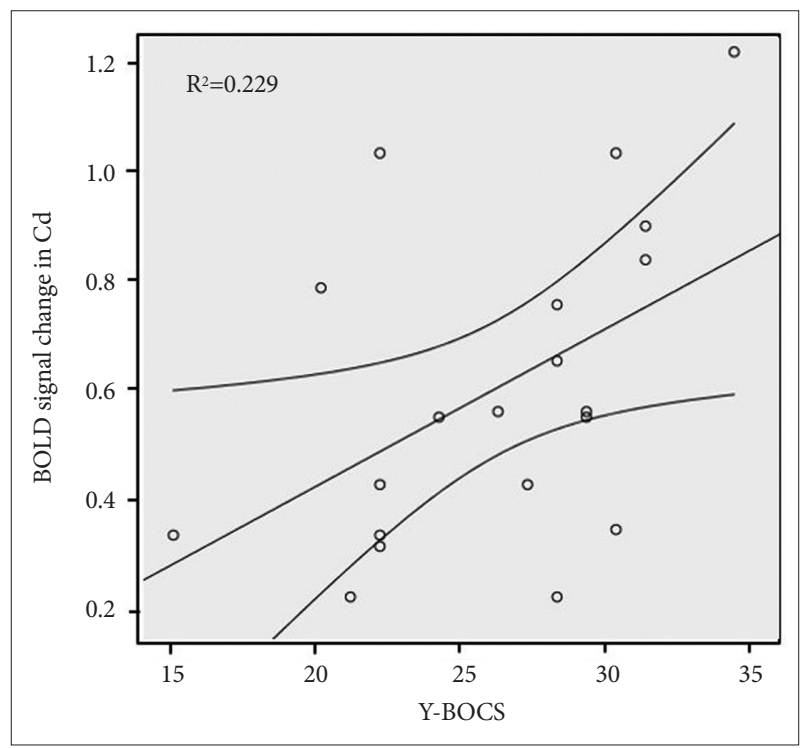

Figure 3. The correlation between the Y-BOCS scores and BOLD signal changes of the caudate $(C d)$ in patients with OCD $(r=0.478$, $p=0.033$ ). The curved line bands mean $95 \%$ confidence intervals. OCD: obsessive-compulsive disorder, Y-BOCS: Yale-Brown Obsessive-Compulsive Scale.

\section{Regional volume difference}

Figure 5 shows volume differences of the GM and WM in patients with OCD vs. healthy controls, in which the highlighted brain areas showing significantly increased brain volumes are summarized in Table 4. Patients with OCD showed significantly higher $\mathrm{GM}$ volumes in the cerebellum $(\mathrm{Cb})$, DLPFC, orbitofrontal cortex (OFC), hippocampus (Hip), Cd and ITG (FWE $\mathrm{p}<0.05$, excluded 30 voxels). However, higher WM volume was observed in the DLPFC only in OCD patients relative to healthy control. It should be noted that remarkable volume reduction of either of GM or WM was not observed in OCD patients. 

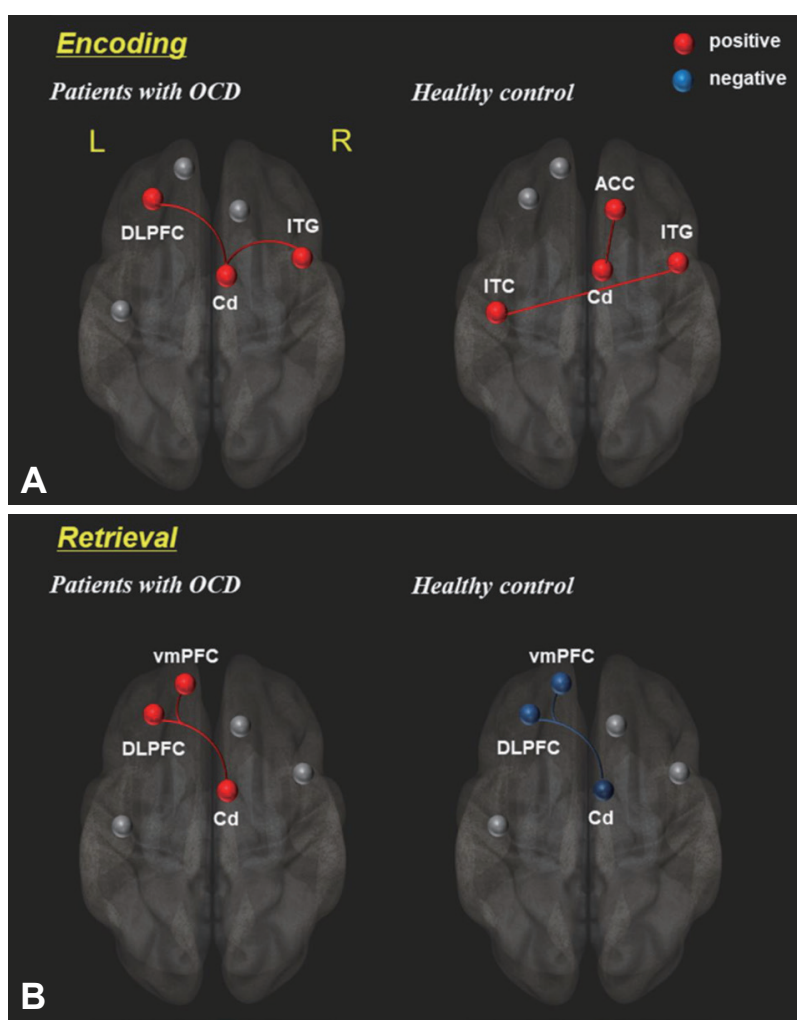

Figure 4. Differential brain connectivity patterns between OCD patients and healthy controls during memory task: $(A)$ encoding epoch and (B) retrieval epoch. Red lines represent increased functional connectivity strength and blue lines represent decreased functional connectivity strength (uncorrected $p<0.005$ ). Details are described in Table 3. DLPFC: dorsolateral prefrontal cortex, ITG: inferior temporal gyrus, Cd: caudate, ACC: anterior cingulate cortex, vmPFC: ventromedial prefrontal cortex, L: left, R: right.

\section{Correlation between GM volume change and symptom severity}

Figure 6A shows a positive correlation between GM volume change of the left OFC and Y-BOCS scores in OCD patients $(\mathrm{r}=0.525, \mathrm{p}=0.017)$. Also, the regions of left OFC $(\mathrm{r}=0.533$, $\mathrm{p}=0.016)$ and right $\mathrm{Cd}(\mathrm{r}=0.458, \mathrm{p}=0.042)$ were positively correlated with HAMA scores, respectively. Pearson's correlation coefficients were satisfied with the critical value as $\mathrm{r}=0.444$ at $\mathrm{p}=0.05$ (two-tailed test).

\section{DISCUSSION}

Numerous conventional studies ${ }^{3,5,12,15}$ have been reported the structural and/or functional abnormalities in memory tasks in patients with OCD. In the current study, we utilized the multimodal combination of neuro-functional activity, functional connectivity and morphometry to evaluate the brain structural and functional abnormalities in connection with memory processing impairment and OCD symptom severity. Here, we assumed that OCD symptoms are probably associated with morphological deficits and abnormal

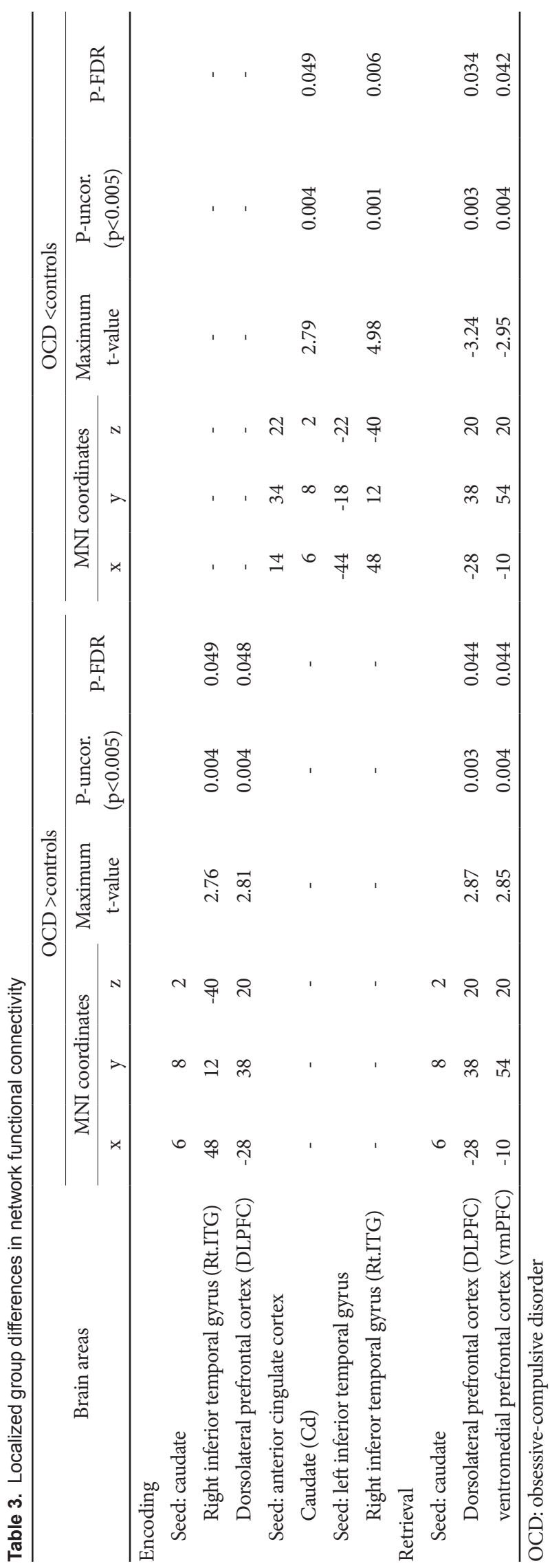


brain activation and neurofunctional connectivity. In this study, patient with OCD showed significantly higher activities in the Lt./Rt. ITG, vmPFC, DLPFC and ACC during the memory encoding epoch, while only the $\mathrm{Cd}$ was markedly activated during the retrieval epoch in memory task (Figure 2 and Table 2). In addition, the functional connectivity patterns of OCD patients were significantly different from those of healthy controls. During the encoding period, OCD patients showed positive connectivity of DLPFC, Cd and Rt. ITG, whereas healthy controls showed different patterns of
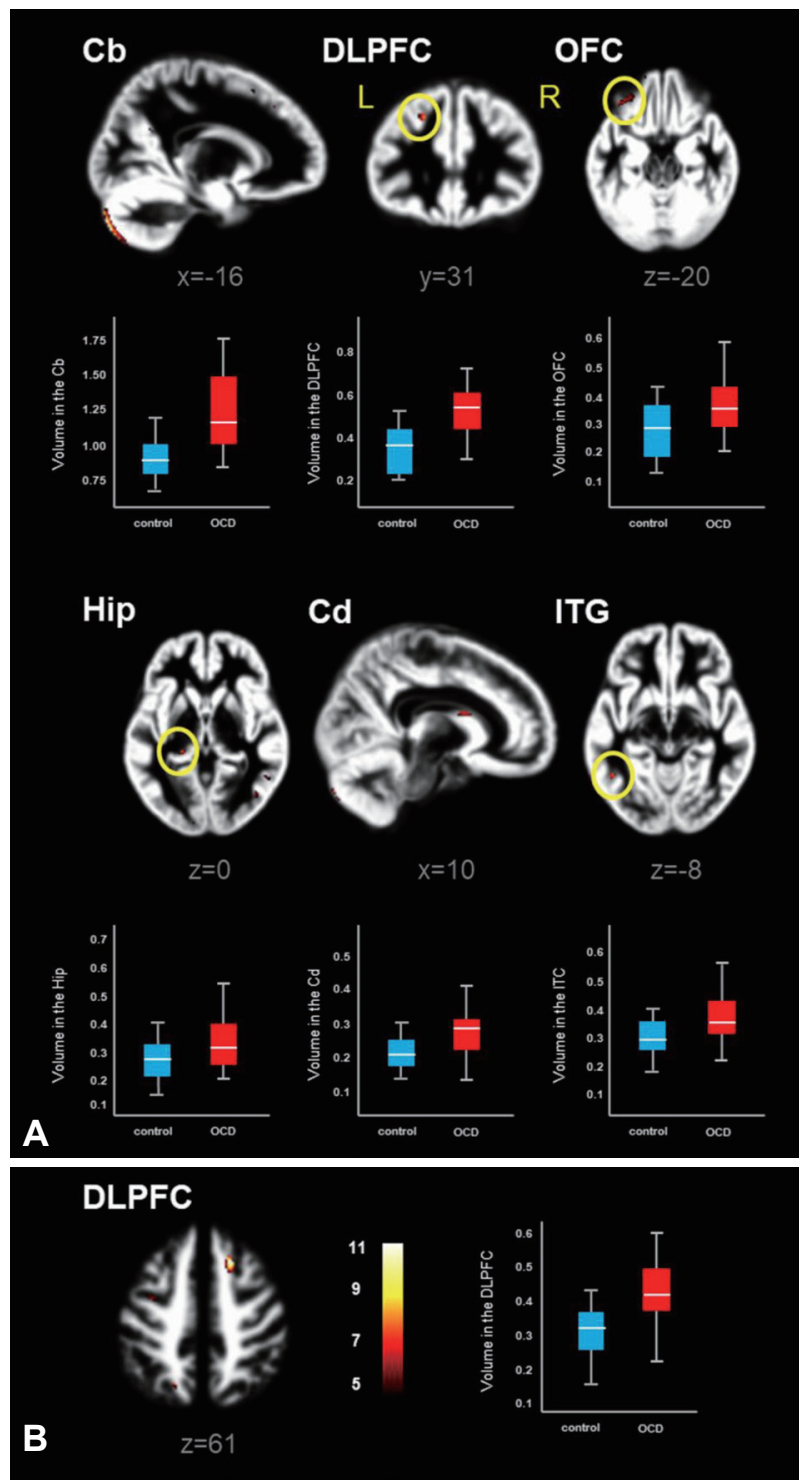

Figure 5. Brain areas showing significantly increased gray matter (A) and white matter $(B)$ volumes in patients with OCD relative to healthy controls, which resulting from two-sample t-test (FEW, $p<0.05$ ). Box-plots show the medians, interquartile ranges, and highest and lowest values for regional volumes $(\mathrm{mL})$ of the following brain areas. $\mathrm{Cb}$ : cerebellum, DLPFC: dorsolateral prefrontal cortex, OFC: orbitofrontal cortex, Hip: hippocampus, Cd: caudate, ITG: inferior temporal gyrus, L: left, R: right. the positive connectivity in the Cd-ACC and Rt. ITG-Lt. ITG. During the retrieval period, OCD patients showed positive connectivity composing of Cd, DLPFC and vmPFC. On the contrary, healthy controls showed negative connectivity in the same areas (Figure 4, Table 3). As for the volumetic comparison between the two groups, OCD patients showed significantly increased volumes of GM (Cb, DLPFC, OFC, Hip, Cd and ITG) and WM (DLPFC) (Figure 5, Table 4). Here, it is important to note that abnormal brain activation of $\mathrm{Cd}$ and volumetric deformation of OFC and Cd are significantly correlated with clinical symptom severities in OCD patients (Figures 3 and 6).

It is well known that OCD symptoms arise from the abnormal neural activity in the cortico-striato-thalamocortical (CSTC) circuitry which is a network involving ACC/vmPFC (affective and reward processing), DLPFC (working memory and executive function), OFC (motor and response inhibition), striatum and thalamus. ${ }^{17-20}$ Our findings shown in Figure 2A, 4 and 5 support the fact that the CSTC pathway is closely linked with the pathogenesis of OCD. Together with predominantly increased brain activities in vmPFC, DLPFC and ACC, the differential functional connectivities of $\mathrm{Cd}$ DLPFC (in OCD patient) and Cd - ACC (in healthy control) are presumably associated with functional abnormality in the CSTC circuit in OCD patients. Among the brain areas mentioned above, the DLPFC and OFC showed higher volumes in OCD patients relative to healthy controls. Many other morphometric studies ${ }^{12,21,22}$ have reported that altered volume difference of DLPFC in OCD patients is a pivotal evidence for the OCD symptoms.

The temporal lobe consists of substructures that are involved in the role of declarative or long-term memory function. Patients with OCD showed abnormal activity in the temporal areas during memory performance. ${ }^{15,23}$ Especially, memory encoding and maintenance processes are implemented through modulation of the inferior temporal activity by the prefrontal cortex. ${ }^{24}$ Moreover, morphometric studies $^{13,25}$ revealed the differential brain volumes and thickness in ITG between OCD patients and healthy controls. In our current study, remarked abnormalities of the brain activation and functional connectivity were observed in ITC during the memory encoding performance in OCD (Figures $2 \mathrm{~A}$ and $4 \mathrm{~A})$. In addition, an increase of the GM volume of the ITG was observed in this study (Figure 5A). These findings may be connected with the evidence that OCD symptoms contribute abnormal neuronal mechanism on the processing of memory modulation and integration.

In comparison with the memory encoding, the memory retrieval step showed different activation pattern (Figure 2B) and functional connectivity (Figure 4B) in OCD patients. Also, the 
patients show the increased GM volume of Cd (Figure 5A). In this area, both of the BOLD signal changes (Figure 3) and GM volume changes (Figure $6 \mathrm{C}$ ) are positively correlated with the OCD symptom severities of Y-BOCS and HAMA, respectively. The Cd plays a role in procedural learning and memory retrieval, and its impairment may lead to abnormal processing of behavioral sequences generated by the frontal subcortical circuits that have been hypothetically existed in OCD. ${ }^{26-30}$ There are many other studies ${ }^{31-35}$ demonstrating that alteration of $\mathrm{Cd}$ volume and abnormal functional activation are associat- ed with symptom severity in OCD. Also, it was reported that continuous psychiatric symptoms more than five years give rise to the increased brain activation and abnormal functional connectivity, inducing the poor memory performance. ${ }^{8}$

Another interesting finding in our study is that patients with OCD showed increased GM volumes in the Hip and OFC (Figure 5A). Hippocampal region and OFC play an essential role in memory encoding and retrieval. ${ }^{15,36}$ Especially, the $\mathrm{OFC}$ is a central region for emotion and social regulation, which is specifically vulnerable to stress, leading to brain vol-

Table 4. Localized GM and WM volume differences between patents with $\mathrm{OCD}$ and Health controls (FWE, $p<0.05, t=5.37)$

\begin{tabular}{|c|c|c|c|c|c|}
\hline \multirow{2}{*}{ Brain areas } & \multirow{2}{*}{$\begin{array}{c}\text { Maximum } \\
\text { t-value }\end{array}$} & \multicolumn{3}{|c|}{ MNI coordinates } & \multirow{2}{*}{$\begin{array}{c}\text { Voxels in } \\
\text { cluster }\end{array}$} \\
\hline & & $\mathrm{x}$ & $\mathrm{y}$ & $\mathrm{z}$ & \\
\hline \multicolumn{6}{|l|}{ GM volume difference } \\
\hline \multicolumn{6}{|l|}{ OCD $>$ control } \\
\hline Cerebellum (Cb) & 10.16 & -16 & -90 & -43 & 434 \\
\hline Inferior parietal conrtex (IPC) & 7.68 & -39 & -50 & 40 & 120 \\
\hline Dosolateral prefrontal cortex (DLPFC) & 6.41 & -18 & 31 & 40 & 63 \\
\hline Orbitofrontal cortex (OFC) & 6.26 & -38 & 37 & -20 & 137 \\
\hline Hippocampus (Hi) & 6.20 & -21 & -31 & 0 & 175 \\
\hline Middle temporal cortex (MTC) & 5.85 & -51 & -41 & 12 & 220 \\
\hline Caudate $(\mathrm{Cd})$ & 5.72 & 10 & 6 & 18 & 87 \\
\hline Inferior temporal conrtex (ITC) & 5.83 & -47 & -54 & -8 & 80 \\
\hline \multicolumn{6}{|l|}{ OCD $<$ control } \\
\hline \multicolumn{6}{|l|}{ None } \\
\hline \multicolumn{6}{|l|}{ WM volume difference } \\
\hline \multicolumn{6}{|l|}{ OCD $>$ control } \\
\hline Dosolateral prefrontal cortex (DLPFC) & 5.94 & -20 & -12 & 61 & 163 \\
\hline \multicolumn{6}{|l|}{ OCD $<$ control } \\
\hline None & & & & & \\
\hline
\end{tabular}

OCD: obsessive-compulsive disorder, FWE: family-wise error
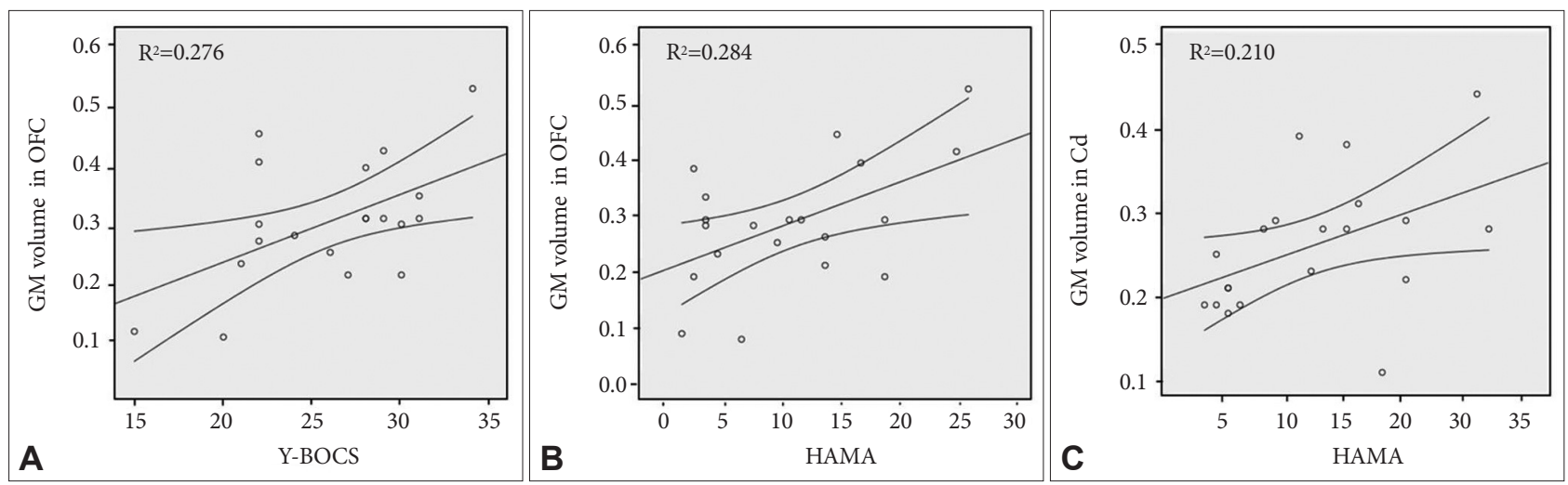

Figure 6. The correlation of the symptom severity and gray matter (GM) volume changes in patients with OCD: (A) Y-BOCS scores vs. GM volumes in the orbitofrontal cortex (OFC), (B) HAMA scores vs. GM volumes in OFC and (C) HAMA scores vs. GM volumes in the caudate (Cd). The curved line bands mean 95\% confidence intervals. OCD: obsessive-compulsive disorder, Y-BOCS: Yale-Brown Obsessive Compulsive Scale, HAMA: Hamilton Anxiety Scale. 
ume change. ${ }^{37,38}$ In addition, correlations were found between the volumes of the OFC and Y-BOCS. ${ }^{39}$ Consequently, such neuroscientific findings support the evidence that the OFC in OCD is associated with memory dysfunction and regional volume changes. The brain volume change and its correlation with psychiatric symptoms in OFC are confirmed in our study (Figure 6A and B).

In the present study, GM volumetric change of the $\mathrm{Cb}$ observed in patients with OCD seems to be associated with its psychopathology (Figure 5A). A meta-analysis study ${ }^{21}$ reported that inhibitory controls affect the decreased brain activation and increased GM volume of $\mathrm{Cb}$ in patients with OCD.

There are several limitations in our study. First, the memory accuracy was not measured and its correlation with brain activation was not analyzed. Future studies are needed to clarify this issue. Secondly, the influence of medication on brain functional and morphometric changes was not excluded. To compensate this week point, further studies including pharmaceutical effect and treatment duration are needed. Thirdly, we performed only the task-induced functional connectivity study. Therefore, this study provides limited information on how the specific brain regions in a network cooperate in doing a memory cognitive task. A combined study with resting-state functional connectivity would be recommended to aid our understanding of the neural mechanism on the OCD.

In conclusion, we believe that the combined use of the neuro-functional activity and local functional connectivity as well as morphometric study will be helpful to understand the neuroanatomical and functional mechanism on abnormal memory processing in connection with OCD symptom severity in patients with OCD. Also, the functional and morphological profiles of Cd should be emphasized for the evaluation of the abnormal processing in memory in connection with OCD symptoms.

\section{Acknowledgments}

This work was supported by National Research Foundation (NRF) grants funded by Korea government (MSIT) (2018R1A2B2006260) and Chonnam National University (CNU) Research Fund for CNU Research Distinguished professor (2017-2022), and Fund of Biomedical Research Institute of Jeonbuk National University Hospital.

\section{Conflicts of Interest}

The authors have no potential conflicts of interest to disclose.

\section{Author Contributions}

Conceptualization: Shin-Eui Park, Jong-Chul Yang, Gwang-Woo Jeong. Data curation: Shin-Eui Park, Jong-Chul Yang. Formal analysis: Shin-Eui Park. Funding acquisition: Jong-Chul Yang, Gwang-Woo Jeong. Investigation: Shin-Eui Park, Gwang-Woo Jeong. Methodology: Shin-Eui Park, Byeong-Chae Kim, Gwang-Woo Jeong. Project administration: GwangWoo Jeong. Resources: Jong-Chul Yang. Software: Shin-Eui Park. Supervision: Jong-Chul Yang, Gwang-Woo Jeong. Validation: Byeong-Chae Kim, Gwang-Woo Jeong. Visualization: Shin-Eui Park. Writing_original draft:
Shin-Eui Park. Writing_review \& editing: Jong-Chul Yang, Gwang-Woo Jeong.

\section{ORCID iDs}

Shin-Eui Park

Byeong-Chae Kim

https://orcid.org/0000-0002-4718-8691

Jong-Chul Yang

https://orcid.org/0000-0001-6827-6730

Gwang-Woo Jeong

https://orcid.org/0000-0002-0621-3168

\section{REFERENCES}

1. Goodman WK, Grice DE, Lapidus KA, Coffey BJ. Obsessive-compulsive disorder. Psychiatr Clin North Am 2014;37:257-267.

2. İnanç L, Altıntaş M. Are mentalizing abilities and insight related to the severity of obsessive-compulsive disorder. Psychiatry Investig 2018;15: 843-851.

3. Nakao T, Nakagawa A, Nakatani E, Nabeyama M, Sanematsu H, Yoshiura T, et al. Working memory dysfunction in obsessive-compulsive disorder: a neuropsychological and functional MRI study. J Psychiatr Res 2009;43:784-791.

4. van den Heuvel OA, Veltman DJ, Groenewegen HJ, Cath DC, van Balkom AJ, van Hartskamp J, et al. Frontal-striatal dysfunction during planning in obsessive-compulsive disorder. Arch Gen Psychiatry 2005;62:301-309.

5. Deckersbach T, Otto MW, Savage CR, L Baer, Jenike MA. The relationship between semantic organization and memory in obsessive-compulsive disorder. Psychother Psychosom 2000;69:101-107.

6. Savage CR, Deckersbach T, Wilhelm S, Rauch SL, Baer L, Reid T, et al. Strategic processing and episodic memory impairment in obsessive compulsive disorder. Neuropsychology 2000;14:141-151.

7. Moon CM, Kim BC, Jeong GW. Associations of neurofunctional, morphometric and metabolic abnormalities with clinical symptom severity and recognition deficit in obsessive-compulsive disorder. J Affect Disord 2018;227:603-612.

8. Heinzel S, Kaufmann C, Grützmann R, Hummel R, Klawohn J, Riesel A, et al. Neural correlates of working memory deficits and associations to response inhibition in obsessive compulsive disorder. Neuroimage Clin 2017;17:426-434.

9. de Vries FE, de Wit SJ, van den Heuvel OA, Veltman DJ, Cath DC, van Balkom AJ, et al. Cognitive control networks in OCD: a resting-state connectivity study in unmedicated patients with obsessive-compulsive disorder and their unaffected relatives. World J Biol Psychiatry 2019;20: 230-242.

10. Park J, Kim T, Kim M, Lee TY, Kwon JS. Functional connectivity of the striatum as a neural correlate of symptom severity in patient with obsessive-compulsive disorder. Psychiatry Investig 2020;17:87-95.

11. Yun JY, Jang JH, Jung WH, Shin NY, Kim SN, Hwang JY, Kwon JS. Executive dysfunction in obsessive-compulsive disorder and anterior cingulate-based resting state functional connectivity. Psychiatry Investig 2017;14:333-343.

12. Park SE, Jeong GW. Cerebral white matter volume changes in patients with obsessive-compulsive disorder: Voxel-based morphometry. Psychiatry Clin Neurosci 2015;69:717-723.

13. Kühn S, Kaufmann C, Simon D, Endrass T, Gallinat J, Kathmann N. Reduced thickness of anterior cingulate cortex in obsessive-compulsive disorder. Cortex 2013;49:2178-2185.

14. American Psychiatric Association. American Psychiatric Association Diagnostic and Statistical Manual of Mental Disorders (4th Ed., Text Rev.). Washington, DC: American Psychiatric Publishing; 2000.

15. Park SE, Yang JC, Jeong GW. Neuroanatomical assessment of the impact of negative emotion on implicit memory in patients with obsessive compulsive disorder. Acta Neuropsychiatr 2016;28:206-213.

16. Behzadi Y, Restom K, Liau J, Liu TT. A component based noise correction method (CompCor) for BOLD and perfusion based fMRI. NeuroImage 2007;37:90-101. 
17. Guimond S, Hawco C, Lepage M. Prefrontal activity and impaired memory encoding strategies in schizophrenia. J Psychiatr Res 2017;91: 64-73.

18. Brod G, Shing YL. Specifying the role of the ventromedial prefrontal cortex in memory formation. Neuropsychologia 2018;111:8-15.

19. Roy M, Harvey PO, Berlim MT, Mamdani F, Beaulieu MM, Turecki G, et al. Medial prefrontal cortex activity during memory encoding of pictures and its relation to symptomatic improvement after citalopram treatment in patients with major depression. J Psychiatry Neurosci 2010; 35:152-162.

20. Milad MR, Rauch SL. Obsessive-compulsive disorder: beyond segregated cortico-striatal pathways. Trends Cogn Sci 2012;16:43-51.

21. Norman LJ, Carlisi C, Lukito S, Hart H, Mataix-Cols D, Radua J, et al, Structural and functional brain abnormalities in attention-deficit/hyperactivity disorder and obsessive-compulsive disorder: a comparative meta-analysis. JAMA Psychiatry 2016;73:815-825.

22. Peng Z, Lui SS, Cheung EF, Jin Z, Miao GD, Jing J, et al. Brain structural abnormalities in obsessive-compulsive disorder: converging evidence from white matter and grey matter. Asian J Psychiatr 2012;5: 290-296.

23. Olson CA, Hale LR, Hamilton N, Powell JN, Martin LE, Savage CR. Altered source memory retrieval is associated with pathological doubt in obsessive-compulsive disorder. Behav Brain Res 2016;296:53-60.

24. Ranganath C, DeGutis J, D’Esposito M. Category-specific modulation of inferior temporal activity during working memory encoding and maintenance. Brain Res Cogn Brain Res 2004;20:37-45.

25. Tinelli E, Francia A, Quartuccio EM, Morreale M, Contessa GM, Pascucci $S$, et al. Structural brain MR imaging changes associated with obsessive-compulsive disorder in patients with multiple sclerosis. AJNR Am J Neuroradiol 2013;34:305-309.

26. Bastin C, Feyers D, Majerus S, Balteau E, Degueldre C, Luxen A, et al. The neural substrates of memory suppression: a FMRI exploration of directed forgetting. PLoS One 2012;7:e29905.

27. Wiggs CL, Weisberg J, Martin A. Neural correlates of semantic and episodic memory retrieval. Neuropsychologia 1999;37:102-118.

28. Suñol M, Contreras-Rodríguez O, Macià D, Martínez-Vilavella G, Martínez-Zalacaín I, Subirà M, et al. Brain structural correlates of sub- clinical obsessive-compulsive symptoms in healthy children. J Am Acad Child Adolesc Psychiatry 2018;57:41-47.

29. Graybiel AM, Rauch SL. Toward a neurobiology of obsessive-compulsive disorder. Neuron 2000;28:343-347.

30. Saxena S, Rauch SL. Functional neuroimaging and the neuroanatomy of obsessive-compulsive disorder. Psychiatr Clin North Am 2000;23: 563-586.

31. Scarone S, Colombo C, Livian S, Abbruzzese M, Ronchi P, Locatelli M, et al. Increased right caudate nucleus size in obsessive-compulsive disorder: detection with magnetic resonance imaging. Psychiatry Res 1992;45:115-121.

32. Robinson D, Wu H, Munne RA, Alvir JM, Lerner G, Koreen A, et al. Reduced caudate nucleus volume in obsessive-compulsive disorder. Arch Gen Psychiatry 1995;52:393-398.

33. Breiter HC, Rauch SL, Kwong KK, Weisskoff RM, Kennedy DN, Kendrick $\mathrm{AD}$, et al. Functional magnetic resonance imaging of symptom provocation in obsessive-compulsive disorder. Arch Gen Psychiatry 1996;53:595-606.

34. Simon D, Kaufmann C, Müsch K, Kischkel E, Kathmann N. Frontostriato-limbic hyperactivation in obsessive-compulsive disorder during individually tailored symptom provocation. Psychophysiology 2010;47:728-738.

35. McGuire PK, Bench CJ, Frith CD, Marks IM, Frackowiak RS, Dolan RJ. Functional anatomy of obsessive-compulsive phenomena. Br J Psychiatry 1994;164:459-468.

36. Moon CM, Jeong GW. Abnormalities in gray and white matter volumes associated with explicit memory dysfunction in patients with generalized anxiety disorder. Acta Radiol 2017;58:353-361.

37. Rempel-Clower NL. Role of orbitofrontal cortex connections in emotion. Ann N Y Acad Sci 2007;1121:72-86.

38. Toga AW, Thompson PM, Sowell ER. Mapping brain maturation. Trends Neurosci 2006;29:148-159.

39. Jayarajan RN, Agarwal SM, Viswanath B, Kalmady SV, Venkatasubramanian G, Shoba Srinath S, et al. A voxel based morphometry study of brain gray matter volumes in Juvenile obsessive compulsive disorder. J Can Acad Child Adolesc Psychiatry 2015;24:84-91. 